\section{Estilo de apego adulto y creencias en relación a masculinidad $y$ femineidad en hombres agresores de su pareja íntima}

Adult attachment style and beliefs in relation to masculinity and femininity in men inflicting violence to their partners

\section{JAVIer BARRÍA-MuÑOZ*}

\section{Resumen}

Se exploraron dos constructos teóricos que recientemente están siendo estudiados en lberoamérica en muestras de hombres agresores, como son: los estilos de apego y de creencias en relación con la masculinidad y la femineidad, en una muestra de 732 agresores y un grupo control de 100 sujetos no

Escuela de Trabajo Social, Pontificia Universidad Católica de Valparaíso, Chile. Correo electrónico: javier.barria@ucv.cl maltratadores. El principal hallazgo en el grupo es la predominancia de estilos de apego ansiosos/preocupados y temerosos/desorganizados entre los maltratadores. Se encontraron, además, diferencias significativas entre agresores y no maltratadores en relación con las creencias misóginas y machistas sobre violencia y género. No obstante lo anterior, Chile, en comparación con otros países, gozaría de mayor equidad de género, que contradice investigaciones que asumen el predominio del machismo como explicación de la violencia de pareja.

Palabras clave: agresores de pareja, apego adulto, creencias en relación a femineidad y masculinidad, hombres agresores.

\section{Abstract}

Attachment styles and beliefs about masculinity and femininity were explored in of sample of 732 offenders and 100 control subjects did not abusive group: two theoretical constructs recently being studied in samples of male aggressors, as are explored. The main findings are that there is a predominance of styles of anxious/worried and fearful/disorganized attachment among batterers, and significant differences between aggressors and not abusive in relation to the misogynistic and sexist beliefs about violence and gender were found. However, Chile, compared to other countries, would be in a place of greater tolerance of gender that contradicts research assume the prevalence of machismo as an explanation for intimate partner violence.

Key words: partner violence against women, adult attachment, beliefs about femininity and masculinity, male aggressors. 


\section{Introducción}

El objetivo de la investigación fue indagar en torno a dos constructos que recientemente se están estudiando en contextos iberoamericanos (España y Chile) en muestras de maltratadores (Aguilera 2004; Barría 2013), como son: los estilos de apego y las creencias en relación a la masculinidad y la feminidad, que constituirían rasgos de personalidad adquiridos por la socialización temprana y que, a posteriori, configuran la identidad de género. Ambos constructos constituyen, todavía en el presente, vacíos conceptuales sobre cómo intervienen (en tanto variables mediadoras) en las reacciones agresivas de los maltratadores (Aguilera 2004; Barría 2013; Loinaz et al. 2012). La hipótesis principal del estudio es que estos dos constructos pueden contribuir a configurar una teoría unificadora de la violencia de género, que sea complementaria con las actuales teorías psicológicas y etiológicas para la intervención con hombres maltratadores (Goldstein y Higgins-D'Alessandro 2000; DeKeseredy y Dragiewicz 2007).

En la literatura hay abundantes referencias sobre las características de los maltratadores como sujetos disfóricos, volátiles o emocionalmente inestables (Holtzworth-Munroe y Stuart 1994; Dutton et al. 1997; Dutton y Haring 1999). Estos estudios muestran la importancia de investigar el componente emocional en la modulación de la conducta agresiva, sobre todo de los estilos de apego. Paulatinamente, estos rasgos de personalidad están siendo asociados por algunos autores como Dutton (2006) al maltrato de la pareja, el que, a su vez, puede vincularse a una protesta contra la figura de apego dada por las amenazas de separación o abandono (Dutton 2006; Loinaz et al. 2012).

\subsection{Apego y hombres agresores de su pareja íntima}

La controversia que sigue pendiente es que el apego no explica la conducta agresiva, sino que sería un constructo útil para la investigación de mecanismos psicológicos, presente, en cuanto variable interviniente, entre los modos de relación del agresor y sus diferentes déficits de empatía o tendencia a la impulsividad (Babcock et al. 2000; Ansbro 2008).

Deacuerdo con la teoría delapego, los individuos internalizan modelos de cuidado de un otro significativo. Se cree que dichos modelos son resistentes al cambio y relativamente estables, y que continúan en la edad adulta y juegan un papel en la vida romántica, en la crianza de los hijos, y en la relación violenta con la pareja (Robinson 2005; Fonagy et al. 2010). Es así como los adultos con estilo de apego seguro han sido caracterizados como emocionalmente más positivos que los adultos inseguros y más confiados, quienes pueden ver a los otros como más dignos de confianza, fiables y con buenas intenciones. Además, éstos se ven (a sí mismos) como amables, dignos, agradables y fáciles de conocer (Goldstein y HigginsD’Alessandro 2000).

El estilo de apego seguro se relaciona positivamente con la satisfacción marital y una comunicación más constructiva. Los adultos con estilo de apego ansioso-preocupado tienden a tener dificultades en la interacción social con otras personas. Debido al uso ineficiente de estrategias de afrontamiento, es menos probable que se autoperciban como agradables y apreciados, y tienden a ver a otros como menos bien intencionados (HoltzworthMunroe et al. 1997). 
Dutton (1999) fue el primero en mencionar, en su hipótesis sobre la personalidad abusiva del maltratador, que los cónyuges violentos tienden hacia el estilo de apego ansioso-ambivalente (Dutton et al. 1997; Dutton 2006). Estos adultos muestran temor y miedo de perder a su cónyuge. En la actualidad, existe consenso en la literatura acerca de que el estilo de apego presente entre los maltratadores es el inseguro o ambivalente o desorganizado (Babcock et al. 2000; Dutton 2006; Loinaz et al. 2012).

Por ejemplo, Babcock et al. (2000) examinaron las diferencias que los hombres violentos establecían en sus relaciones, tomando como variables del estudio los estilos de apego y la regulación emocional. Encontraron que los hombres violentos desean cercanía con su pareja, pero también distancia y libertad, sin embargo, no quieren lo mismo para sus esposas, y es probable que les dificulte ser abandonados o abandonar a su pareja (Babcock et al. 2000). Estos autores plantean que un estilo de apego ambivalente, en conjunto con la conducta ansiosa del hombre, podría llegar a ser una fuente de violencia cuando éste empieza a tener miedo por la pérdida de su relación de pareja. Más tarde, Robinson (2005) menciona que el apego inseguro no explica la conducta violenta, pero permite entender los procesos psicológicos y emocionales presentes, tales como déficits cognitivos y reactancia emocional, además de cierta tendencia a la impulsividad.

\subsection{Creencias en relación a la masculinidad y la femineidad en hombres agresores de su pareja íntima}

Existe abundante información en Latinoamérica que indica que la violencia de pareja está asociada a pautas de crianza traumáticas de las figuras parentales hacia sus hijos, tales como: haber sufrido maltrato en su proceso de socialización (Stith et al. 2000; Rey y César 2008), o haber tenido historias generacionales de violencia doméstica (Pears y Capaldi 2001), padres que abusan de sustancias psicoactivas (Barría y Macchiavello 2012a), o padres con intolerancia y nula comunicación (Escudero et al. 2005).

Hechos estos alcances, este artículo asume como hipótesis que la agresión de pareja es un hecho multicausal, producto de toda una dinámica que involucra la construcción social de la afectividad, los estilos de apego y las creencias que se asumen en relación con la masculinidad y la femineidad, que se integrarían como parte de los rasgos de personalidad en el maltratador (Moya et al. 2002; Aguilera 2004; Gil-González 2005; Gondolf 2007).

Los últimos estudios en masculinidades han evidenciado que el género es una construcción social de la identidad (independiente de la biología), que está determinada en gran medida por las creencias que se asumen sobre roles de género como diferencias entre hombres y mujeres (Fernández y Vergara 1998; De Keijzer 2003). Sin embargo, existe evidencia de que los varones, en la construcción de su masculinidad, sufren una mutilación de ciertas características de su personalidad, las cuales culturalmente son asociadas como femeninas (Bosch et al. 2014). En esta mutilación existe un abuso del concepto de machismo (Aguilera 2004), como representación o caricatura de la cultura patriarcal que implica que el varón asuma un conjunto de rasgos como propios de la masculinidad, esto es, no ser femenino, lo que implica cierta libertad sexual, ser viril y no 
desarrollar intimidad con los varones (Bonino 2003), tener poder y ser admirado (Garda 2004), y demostrar uso de la fuerza física, ser audaz, correr riesgos y siempre tener la razón (De Keijzer 2003).

\section{Propuesta investigativa}

La investigación explora estos dos constructos que sólo recientemente están siendo explorados en la violencia masculina: los estilos de apego adulto y las creencias en relación a la masculinidad y la femineidad. Pretende, además, plantear la hipótesis sobre la importancia de estos modelos teóricos como co-variantes, y con los cuales se puede ir configurando una teoría unificadora sobre la violencia de género desde una perspectiva de las relaciones vinculares y las creencias asociadas a la masculinidad y la femineidad ya referidas.

\section{Método}

\subsection{Diseño}

Se diseñó una investigación no experimental y transeccional, de carácter descriptivo y correlacional.

\subsubsection{Participantes}

La muestra fue de carácter intencional e incluyó a la totalidad de la cohorte atendida entre los meses de enero de 2012 a enero de 2013, cuyos usuarios son parte del programa "Hombres por una vida sin violencia" del Servicio Nacional de la Mujer (SERNAM), presente en las ciudades de Santiago, Viña del Mar, Rancagua, Talca, Puerto Montt, Arica, Antofagasta, Copiapó, La Serena, Iquique, Valdivia, Temuco, Concepción, Coihaique y Punta Arenas. La muestra quedó conformada por 832 sujetos que cumplían los requisitos de ser mayores de 18 años. Fueron derivados por violencia física o psicológica desde Fiscalía, Tribunales de Familia, Juzgados de Garantía, Instituciones del Estado, Fuerzas Armadas, instituciones de educación, salud y empresas en general. Además, se incorporaron a la muestra los sujetos ingresados por demanda espontánea que asisten en forma voluntaria al programa, junto con un grupo control de 100 de sujetos sin historia de violencia previa.

\subsubsection{Instrumento}

\subsubsection{Inventario de creencias en relación a la femineidad y la masculinidad (IMAFE) (Lara 1993)}

El inventario de creencias en relación a la femineidad y la masculinidad mide los roles de género a partir de la evaluación de características masculinas y femeninas de la personalidad: machismo v/s sumisión (Gómez et al. 2012). El instrumento está basado en los aspectos más representativos de los roles de género y estereotipos en la cultura. Éste consta de cuatro escalas, de las cuales se identifican seis perfiles: masculino, femenino, machismo, androginia, indiferenciación o sin extremos, y sumisión. El IMAFE incluye cuatro sub-escalas:

a) Masculinidad: mide rasgos pragmáticos o autoafirmativos de masculinidades, tales como: confiado de sí mismo, capaz de tomar decisiones, independiente, analítico, competitivo, racional, reflexivo, con personalidad fuerte, hábil 
para dirigir, seguro de sí mismo, autosuficiente, dispuesto a arriesgarse, valiente, atlético, maduro. Corresponden a los ítems 1, 5, 9, 13, $17,21,25,29,33,37,41,45,49,53,57$.

b) Femineidad: mide la capacidad del sujeto de expresar rasgos psicológicos y afectivos asociados a lo femenino, tales como: sensible a las necesidades de los demás, comprensivo, tierno, afectuoso, cariñoso, con gusto por los niños, dulce, caritativo, de voz suave, compasivo, amigable. Corresponden a los ítems 2, 6, 10, 14, 18, 22, 26, 30, 34, 38, 42 ,46, $50,54,58$.

c) Machismo: mide la expresión de rasgos de dominio y agresión, tales como: agresivo, dominante, autoritario, egoísta, materialista, frío, ambicioso, rudo, enérgico, incomprensivo, individualista, arrogante. Corresponden a los ítems 3, 7, 11, 15, 19, 23, 27, 31, 35, 39, 43, 47, $51,55,59$.

d) Sumisión: mide la capacidad de expresar una masculinidad pasiva o sumisa, con rasgos tales como: indeciso, de personalidad débil, cobarde, sumiso, incapaz de planear, dependiente, influenciable, conformista, tímido, retraído, pasivo, simplista, resignado, inseguro de sí mismo. Corresponden a los ítems 4, 8, 12, 16, 20, 24, 28, 32, 36, 40, 44, 48, 5, 58, 60.

Para obtener las tipologías del IMAFE se calcula mediante la prueba $\mathrm{T}$ de Student el nivel de significación, considerando el máximo valor en 0,05 (un 95\% de confianza), para determinar que existen diferencias significativos entre las polaridades masculino/femenino y machismo/ sumisión. Con ello se obtienen cuatro tipologías: androginia, masculinidad, machismo y misoginia. La escala de 60 ítems fue sometida a prueba de consistencia interna mediante el cálculo del coeficiente Alfa de Cronbach, un coeficiente estandarizado de .78, lo cual indica un buen nivel de consistencia interna.

\subsubsection{Escala de experiencia en relaciones cercanas (ECR-2) (Brenam, Clark y Shaver 1998)}

El ERC tiene 2 escalas: evitación y ansiedad. El primero corresponde a los ítems 1, 5, 7, $9,11,13,17,21,23$, cuya escala invertida corresponde a los ítems 3, 15, 19, 25, 27, 29, $31,33,35)$. El segundo corresponde a los ítems $4,6,8,10,12,14,16,18,20,24,26,28,30$, $32,34,36$, cuya escala invertida corresponde al ítem 22.

A partir de las escalas se establecieron cuatro tipologías: a) apego seguro: baja ansiedad y baja evitación; b) apego preocupado: alta ansiedad y baja evitación; c) apego rechazante: baja ansiedad y alta evitación, y; d) apego temeroso: alta ansiedad y alta evitación (Fraley et al. 2000).

En la pregunta 22 de la escala de ansiedad y en las preguntas $3,15,19,25,27,29,31,33,35$ de la escala de evitación se debe invertir el puntaje que anotó el sujeto en la hoja de respuesta de la siguiente forma: $1=7 ; 2=6 ; 3=5 ; 4=4 ; 5=3$; $6=2 ; 7=1$. Una vez realizadas las inversiones de escala, se calcula cada escala por separado sumando los puntajes de los 18 ítems, como lo sugieren Brennan, et al. (1998).

Los autores no proponen un puntaje general o global, sino escalas dicotomizadas, para luego buscar la tipología que les corresponde. Para facilitar la aplicación masiva del instrumento, 
se fijó el valor subjetivo a las subescalas, estableciendo el percentil 50 como punto de corte, señalado alta (+) o baja (-) ansiedad o evitación. El punto de corte para la validación nacional (Barría 2013) quedó establecido en los 54 puntos para la escala de evitación, y 68 puntos para la escala de ansiedad. La escala de 49 ítems fue sometida a prueba de consistencia interna mediante el cálculo del coeficiente Alfa de Cronbach, obteniéndose un coeficiente estandarizado de .79, lo cual indica un buen nivel de consistencia interna.

\subsubsection{Escala de tácticas de conflicto (CTS-2) (Straus et al. 1996)}

Esta escala mide el grado en que los miembros de una pareja se ven envueltos mutuamente en ataques físicos o psicológicos, así como en el uso del razonamiento y la negociación para resolver sus conflictos. Tiene 78 ítemes: 39 para cada miembro de la pareja (Loinaz 2009; Straus et al. 1996).

EI CTS-2 posee cinco sub-escalas: a) violencia física corresponde a los ítems 7, 9, 17, 45, 53, 21, 27, 33, 37, 43, 61,73; b) coacción (coerción) sexual corresponde a los ítems 15, 51, 63, 19, $47,57,75$; c) agresión psicológica corresponde a los ítems 5, 35, 49, 67, 25, 29, 65, 69; d) daños corresponde a los ítems 11, 74, 33, 31, 41, 55, y; e) negociación corresponde a los ítems 1, 13, 39, 3, 39, 77.

Las escalas mencionadas se dividen, a su vez, en dos sub-escalas: cognitiva y emocional para negociación, y menor o severa para las demás escalas. Consta de 8 alternativas de respuesta. Los valores del 0 al 6 corresponden a la frecuencia con la que puede haber ocurrido una conducta: 1 (una vez), 2 (dos veces), 3 (entre tres y cinco veces), 4 (de seis a diez veces), 5 (de once a veinte veces) y 6 (más de 20 veces). El 7 se refiere a nunca el año pasado, pero sí antes, y el 0 significa nunca ha ocurrido. Las principales puntuaciones de la escala son las siguientes:

a) Frecuencia: número de veces en que ha sucedido una conducta dentro de cada escala en el período de referencia.

b) Prevalencia: presencia o ausencia de la conducta. Se calcula transformando las respuestas 1-7 en 1, y el 0 en 0 . Los ítems no se suman, por lo que la prevalencia para cada sub-escala será 1 o 0 . Para la prevalencia anual el ítem 7 puntuaría 0 (Straus et al. 1996).

c) Severidad de la violencia física: se asigna un valor teórico propuesto por Straus et al. (1996) a cada ítem de las escalas de violencia física: ítems 7, 9, 17, 45 y $53=1$; ítems 27 y $73=3$; ítems 33, 37, 43 y $61=5$, e; ítem $21=8$.

La escala de 39 ítems, correspondientes a las categorías de análisis del maltratador, fue sometida a una prueba de consistencia interna mediante el cálculo del coeficiente Alfa de Cronbach, obteniéndose un coeficiente estandarizado de .91, lo cual indica un alto nivel de consistencia interna.

\subsubsection{Inventario clínico multiaxial de Millon (MCMI-III) (Millon y Davis 1998)}

Consiste en un auto-informe de sólo 175 ítems con un formato verdadero-falso, que evalúa diferentes aspectos de la personalidad a través de 26 escalas. De ellas, 11 corresponden a patrones clínicos de personalidad: esquizoide, 
evitativa, depresiva, dependiente, histriónica, narcisista, antisocial, agresivo-sádica, compulsiva, negativa (pasivo-agresiva) y autodestructiva; 3 corresponden a una patología grave de la personalidad: esquizotípica, límite y paranoide; 7 corresponden a síndromes clínicos: ansiedad, trastorno somatomorfo (histeriforme), bipolaridad, distimia, dependencia al alcohol, abuso de sustancias y trastorno de estrés postraumático, y; 3 corresponden a síndromes clínicos graves: trastorno del pensamiento (psicoticismo), depresión mayor y trastorno delirante. Además, este instrumento dispone de 4 escalas de fiabilidad y validez: validez, sinceridad, deseabilidad y distorsión. Los coeficientes Alfa de Conbrach de consistencia interna de la versión española para las diferentes escalas oscilan, en general, entre .70 y .90 (Millon y Davis 1998).

\subsubsection{Procedimiento y análisis de los datos}

La batería de instrumentos fue aplicada por los equipos técnicos de cada una de las 15 ciudades donde se ejecuta el programa "Hombres por una vida sin violencia", con el consentimiento informado de los sujetos, en el período donde se evalúa al sujeto y se fija su ingreso a una modalidad de intervención psicosocial grupal. El investigador principal confeccionó una base de datos a nivel nacional, y realizó devoluciones de los aspectos psicométricos de los instrumentos a los equipos técnicos de cada sede.

Los análisis estadísticos se realizaron con el programa SPSS versión 20. Se realizaron tres tipos de análisis. Enel primer análisis se realizó un MANOVA (Análisis de la Varianza Multivariante) para establecer diferencias significativas entre los grupos. Adicionalmente, se utilizó la prueba
Tukey para un análisis post hoc. En un segundo análisis, para medir posibles correlaciones en tablas de contingencia, se utilizó la prueba de Chi cuadrado. En un tercer análisis, para medir variables categóricas y variables numéricas, se utilizó la ANOVA de Kruskal-Wallis.

\section{Resultados}

\subsection{Antecedentes sociodemográficos}

El promedio de edad es de 38,7 años, con una desviación estándar de 11,1. La edad de la víctima es de 36 años. Las edades fluctúan entre los 18 a los 78 años. Los tramos de edades de mayor frecuencia son los de 31 a 40 años (33.3\%), y de 41 a 50 años (23.6\%). La muestra es bastante homogénea, ya que el 95.1\% (658) son chilenos, el 2.2\% (16) se reconocen como pueblos originarios (mapuche), y el 2.6\% (12) son extranjeros: peruanos (5), bolivianos (2), españoles (1), italianos (1), cubanos (1) y egipcios (1).

En cuanto a las áreas de ocupación, los maltratadores realizan una amplia variedad de empleos y oficios, predominando las áreas del comercio (22.3\%), servicios (18.6\%) y construcción (12.1\%). En el caso de la víctima, los oficios son dueña de casa (18\%), servicios (aseos, asesora del hogar) (18\%), y comercio (17\%). El nivel de escolaridad se concentra en la enseñanza media completa (28.7\%), incompleta $(13.6 \%)$ y básica.

Sólo un porcentaje reducido (13.6\%) ha presentado conductas antisociales, tales como: beber en la vía pública, conducción bajo la influencia del alcohol, riñas en la vía pública. Sólo el 2,3\% tiene antecedentes delictuales por robo o hurto. 
Respecto a los antecedentes de consumo de alcohol y drogas, el $37.5 \%$ presenta un consumo problemático o abusivo del alcohol, y el 32.2\% reconoce emborracharse todos los días, hasta una vez al mes. El 19.1\% consume drogas, tales como marihuana (3.7\%) y cocaína/ pasta Base (1.0\%). Sólo el $5 \%$ reconoce que ha consumido alcohol o drogas en los momentos de violencia con su pareja.

\subsection{Estilos de apego en relaciones cercanas de maltratadores y no maltratadores}

Los análisis estadísticos se orientan a responder si se pueden discriminar estilos de apego adulto entre muestras de hombres agresores y sin historia de violencia previa, derivados de Tribunales de Familia, Fiscalía, y Voluntarios. También se incluye un análisis complementario donde se evalúa la relación entre nivel de riesgo del agresor y su estilo de apego adulto.

Se efectuaron ocho análisis estadísticos. Primero se estableció que existe asociación entre las variables derivación y estilos de apego. Como vemos en Tabla 1, los estilos de apego se correlacionan con las vías de derivación por la cual ingresan los sujetos al programa "Hombres por una vida sin violencia" $\left(X^{2}(9.424)=20.3\right.$, $\mathrm{p}=.016$ ), existiendo un predominio de los estilos de apegos ansiosos/preocupados $(27.8 \%$ a $39,7 \%)$, y temerosos/desorganizados (25.6\% a $42.3 \%$ ) entre la población de maltratadores, a diferencia de la población de hombres sin historia de violencia previa, en el cual predomina el estilo de apego seguro (66.7\%).

En un segundo análisis se contrastaron las sub-escalas del ERC-2. Se utilizó la MANOVA, obteniéndose como resultado que existen diferencias significativas entre el grupo de hombres sin historia de violencia y los grupos de hombres agresores en la escala de ansiedad $(F(3.83)=8.1, p=.000)$, y no se encontraron diferencias significativas en la escala de evitación $(\mathrm{F}(3.511)=2.2, \mathrm{p}=.08)$.

En un tercer análisis se utilizó la ANOVA cualitativa prueba de Kruskal Wallis (Teruelo 2011) para grupos independientes y aleatorios que proceden de una misma población, pero cuyos datos son categóricos, como es el caso del instrumento ERC-2. Entre los resultados obtenidos se desprende que existen diferencias significativas en los sujetos derivados por demanda espontánea en los estilos de apego desorganizado/temeroso $\left(X^{2}(3.225)=288.08, p=.000\right)$, y en el estilo de apego ansioso existen diferencias significativas entre los sujetos ingresados vía tribunal de familia $\left(X^{2}(3.139)=286.27, p=.000\right)$.

Posteriormente, se analizó la relación entre el nivel de riesgo de los usuarios y los estilos de apego adulto. Como veremos en la Tabla 2 , los estilos de apego y el nivel de riesgo del maltratador están asociados $\left(X^{2}(9.511)=36.5\right.$, $\mathrm{p}=.000$ ). Los grupos de agresores de riesgo alto y extremo están concentrados en los estilos de apego desorganizado (16.2\%) y ansioso (15.1\%). En cambio, los sujetos de riesgo bajo se concentran en el estilo de apego seguro (11.2\%).

En un quinto análisis utilizando las sub-escalas del ERC-2 se estableció mediante el uso de la MANOVA que existen diferencias significativas en la escala de evitación $(F(3.511=8.9, p=.000)$ en los grupos de extremo y alto riesgo. También existen diferencias significativas en la escala de ansiedad $(F(3.514)=4.09, p=.000)$ en el grupo de extremo riesgo. 
Tabla 1. Estilos de apego, creencias en masculinidad y femineidad, vías de derivación

\begin{tabular}{|c|c|c|c|c|c|c|c|c|c|c|}
\hline & \multicolumn{2}{|c|}{$\begin{array}{l}\text { Demanda } \\
\text { espontánea }\end{array}$} & \multicolumn{2}{|c|}{$\begin{array}{c}\text { Tribunales de } \\
\text { familia } \\
\text { (Vif } \\
\text { Psicológico) }\end{array}$} & \multicolumn{2}{|c|}{$\begin{array}{c}\text { Fiscalía } \\
\text { (Vif Físico) }\end{array}$} & \multicolumn{2}{|c|}{$\begin{array}{c}\text { No } \\
\text { maltratadores }\end{array}$} & \multicolumn{2}{|c|}{ TOTALES } \\
\hline andrógino & 73 & $17,2 \%$ & 50 & $11,8 \%$ & 36 & $8,5 \%$ & 39 & $9,2 \%$ & 198 & $46,7 \%$ \\
\hline masculinidad & 29 & $6,8 \%$ & 32 & $7,5 \%$ & 13 & $3,1 \%$ & 10 & $2,4 \%$ & 84 & $19,8 \%$ \\
\hline machismo & 37 & $8,7 \%$ & 14 & $3,3 \%$ & 17 & $4 \%$ & 12 & $2,8 \%$ & 80 & $18,9 \%$ \\
\hline misoginia & 26 & $6,1 \%$ & 25 & $5,9 \%$ & 7 & $1,7 \%$ & 4 & $0,9 \%$ & 62 & $14,6 \%$ \\
\hline TOTALES & 165 & $38,9 \%$ & 121 & $28,5 \%$ & 73 & $17,2 \%$ & 65 & $15,3 \%$ & 424 & $100,0 \%$ \\
\hline \multicolumn{11}{|c|}{$\begin{array}{l}\text { Resultados obtenidos con el IMAFE y el CTS-2 } \\
X^{2}(9, N=513)=91,6, p=.000 ; r(.-328)=-7.8, p=.000\end{array}$} \\
\hline & \multicolumn{2}{|c|}{$\begin{array}{l}\text { Demanda } \\
\text { espontánea }\end{array}$} & \multicolumn{2}{|c|}{$\begin{array}{c}\text { Tribunales de } \\
\text { familia } \\
\text { (Vif } \\
\text { Psicológico) }\end{array}$} & \multicolumn{2}{|c|}{$\begin{array}{c}\text { Fiscalía } \\
\text { (Vif Físico) }\end{array}$} & \multicolumn{2}{|c|}{$\begin{array}{c}\text { No } \\
\text { maltratadores }\end{array}$} & \multicolumn{2}{|c|}{ TOTALES } \\
\hline seguro & 42 & $8,2 \%$ & 25 & $4,9 \%$ & 22 & $4,3 \%$ & 43 & $8,4 \%$ & 132 & $25,7 \%$ \\
\hline evitativo & 33 & $6,4 \%$ & 30 & $5,8 \%$ & 24 & $4,7 \%$ & 8 & $1,6 \%$ & 95 & $18,5 \%$ \\
\hline ansioso & 73 & $14,2 \%$ & 44 & $8,6 \%$ & 22 & $4,3 \%$ & 7 & $1,4 \%$ & 146 & $28,5 \%$ \\
\hline desorganizado & 77 & $15 \%$ & 40 & $7,8 \%$ & 22 & $4,3 \%$ & 1 & $0,2 \%$ & 140 & $27,3 \%$ \\
\hline TOTALES & 225 & $43,9 \%$ & 139 & $27,1 \%$ & 90 & $17,5 \%$ & 59 & $11,5 \%$ & 513 & $100,0 \%$ \\
\hline
\end{tabular}

Fuente: elaboración propia.

Al igual que en el análisis anterior, se utilizó la prueba de ANOVA cualitativa de Kruskal-Wallis. Existen diferencias significativas en los grupos de alto y extremo riesgo en relación con los estilos de apego temeroso $\left(X^{2}(3.124)=25.04\right.$, $p=.000),\left(X^{2}(3.137)=19.3, p=.000\right)$.

\subsection{Creencias en relación a la masculinidad y la feminidad entre maltratadores y no maltratadores}

El sexto análisis estadístico trata de responder si se pueden hacer distinciones entre el tipo de creencias en relación a la masculinidad y la femineidad y las vías de derivación, es decir, si existe una mayor concentración de creencias misóginas y machistas entre los grupos de agresores. Para responder a esta hipótesis se incorporan dos análisis complementarios, uno entre creencias y nivel de riesgo, y otro entre creencias y estilos de apego.

La tabulación del IMAFE sugiere una dicotomización por cada sujeto (individual) entre las escalas masculinidad-femineidad y machismo-sumisión. Por diferencias de puntajes $\mathrm{T}$, el sujeto queda en la categoría que 
está descrita en la Tabla 1, en androginia (alta tolerancia a la femineidad), y masculinidad, machismo y misoginia (mínima tolerancia a la sumisión). Como podemos ver, las creencias sobre masculinidad y feminidad están asociadas con la variable vías de derivación $\left(\mathrm{X}^{2}\right.$ $(9.424)=20.3, p=.004)$ (ver Tabla 1). El mayor porcentaje de machismo (21.3\%) está entre los sujetos de demanda espontánea, seguido por el grupo de Fiscalía (20.2\%), y los grupos misóginos están entre los derivados por Tribunales de Familia (12.7\%) y por demanda espontánea (14.2\%). Es significativo el hecho de que los niveles de androginia en todos los grupos van desde el $52.6 \%$ (demanda espontánea) y el $70.8 \%$ entre quienes no tienen historia de violencia previa.

Con el análisis de la MANOVA se obtiene como resultado que existen diferencias significativas entre el grupo de hombres sin historia de violencia y aquellos derivados desde Tribunales de Familia, y los grupos de Voluntarios y Fiscalía en la escala de masculinidad $(F(3.65)=3.2$, $\mathrm{p}=.021)$. Se encontraron diferencias significativas en los grupos sin violencia previa y derivados de Fiscalía (VIF físico) en la escala de machismo $(\mathrm{F}(3.65)=7.3, \mathrm{p}=.000)$. Existen también diferencias significativas entre el grupo de hombres sin historia de violencia previa y los grupos de agresores en la escala de sumisión (misoginia) $(\mathrm{F}(3.65)=11.2, \mathrm{p}=.000)$. También hay diferencias significativas entre los grupos en la escala de feminidad $(F(3.65)=3.4, p=.017)$, que estaría expresada en el grupo de hombres sin historia de violencia previa.

En la Tabla 2 podemos ver que las creencias sobre masculinidad y femineidad están asociadas al nivel de riesgo de los agresores $\left(X^{2}(9.498)=9.4, p=.000\right.$. Existen diferencias significativas entre los grupos en la escala de masculinidad $(F(3.138)=3.3, \quad p=.019)$ en el grupo de bajo riesgo; en la escala de femineidad $(\mathrm{F}(3.138)=3.8, \mathrm{p}=.009)$ en el grupo de riesgo extremo; en la escala de machismo $(F(3.138)=34.5, p=.000)$ en los grupos de alto y extremo riesgo, y; en sumisión $(F(3.138)=9.5$, $\mathrm{p}=.000$ ) en el grupo de alto y extremo riesgo.

Por último, en la Tabla 3 observamos que los estilos de apego están asociados a las creencias sobre masculinidad y femineidad $\left(X^{2}(9.493)=17.1, p=.047\right)$. Existen diferencias significativas entre el estilo de apego ansioso y las creencias de machismo $(F(3.133)=1.9$, $\mathrm{p}=.0 .15)$, y entre los estilos de apego ansioso y desorganizado y la escala de sumisión $(\mathrm{F} 3.133)=8.1, \mathrm{p}=.000)$.

\section{Discusión}

Los tramos y promedios de edades son similares a los encontrados en los estudios españoles (Echauri et al. 2005; Loinaz et al. 2010). Las diferencias están en la cantidad, variedad y porcentaje de usuarios extranjeros que muestran los estudios en España, en cambio, Chile muestra un grupo homogéneo y principalmente nacional.

\subsection{Creencias sobre masculinidad y femineidad}

Hay que tomar con prudencia las escalas de misoginia y machismo, porque lo que están señalando los distintos ítems del IMAFE es que los maltratadores tienen un esquema rígido de roles de género, y no necesariamente esto se desprende de un odio a la mujer, tal como 
Tabla 2. Nivel de riesgo, estilos de apego y creencias en relación a masculinidad y femineidad

\begin{tabular}{|c|c|c|c|c|c|c|c|c|c|c|}
\hline \multirow[b]{2}{*}{ andrógino } & \multicolumn{2}{|c|}{ Riesgo bajo } & \multicolumn{2}{|c|}{ Riesgo medio } & \multicolumn{2}{|c|}{ Riesgo alto } & \multicolumn{2}{|c|}{ Riesgo extremo } & \multicolumn{2}{|c|}{ TOTALES } \\
\hline & 60 & $12 \%$ & 42 & $8,4 \%$ & 56 & $11,2 \%$ & 55 & $11 \%$ & 213 & $42,8 \%$ \\
\hline masculinidad & 27 & $5,4 \%$ & 35 & $7 \%$ & 25 & $5 \%$ & 25 & $5 \%$ & 112 & $22,5 \%$ \\
\hline machismo & 24 & $4,8 \%$ & 18 & $3,6 \%$ & 29 & $5,8 \%$ & 22 & $4,4 \%$ & 93 & $18,7 \%$ \\
\hline misoginia & 17 & $3,46 \%$ & 22 & $4,4 \%$ & 28 & $5,6 \%$ & 13 & $2,6 \%$ & 80 & $16,1 \%$ \\
\hline TOTALES & 128 & $25,7 \%$ & 117 & $23,5 \%$ & 138 & $27,7 \%$ & 115 & $23,1 \%$ & 498 & $100 \%$ \\
\hline
\end{tabular}

Resultados obtenidos con el IMAFE y el CTS-2

$\mathrm{X}^{2}(9, N=498)=12.2, \mathrm{p}=.20 ; \mathrm{r}(-.004)=.84, \mathrm{p}=.93$

\begin{tabular}{|l|c|c|c|c|c|c|c|c|c|c|}
\hline & \multicolumn{2}{|c|}{ Riesgo bajo } & \multicolumn{2}{|c|}{ Riesgo medio } & \multicolumn{2}{|c|}{ Riesgo alto } & \multicolumn{2}{c|}{ Riesgo extremo } & TOTALES \\
\hline seguro & 57 & $\begin{array}{c}11,2 \\
\%\end{array}$ & 31 & $6,1 \%$ & 30 & $5,9 \%$ & 18 & $3,5 \%$ & 136 & $26,6 \%$ \\
\hline evitativo & 17 & $3,3 \%$ & 22 & $4,3 \%$ & 25 & $4,9 \%$ & 28 & $5,5 \%$ & 92 & $18 \%$ \\
\hline ansioso & 30 & $5,9 \%$ & 42 & $8,2 \%$ & 43 & $8,4 \%$ & 34 & $6,7 \%$ & 149 & $29,2 \%$ \\
\hline desorganizado & 29 & $5,7 \%$ & 22 & $4,3 \%$ & 39 & $7,6 \%$ & 44 & $8,6 \%$ & 134 & $26,2 \%$ \\
\hline TOTALES & 133 & $26 \%$ & 117 & $22,9 \%$ & 137 & $26,8 \%$ & 124 & $24,3 \%$ & 511 & $100 \%$ \\
\hline
\end{tabular}

Resultados obtenidos con el ERC y el CTS-2

$\mathrm{X}^{2}(9, N=511)=36.5, \mathrm{p}=.000 ; \mathrm{r}(.198)=4.5, \mathrm{p}=.000$

Fuente: elaboración propia.

Tabla 3. Estilos de apego adulto y creencias en relación a la masculinidad y la femineidad

\begin{tabular}{|c|c|c|c|c|c|c|c|c|c|}
\hline \multirow[b]{2}{*}{ seguro } & \multicolumn{2}{|c|}{ andrógino } & \multicolumn{2}{|c|}{ masculinidad } & \multicolumn{2}{|c|}{ machismo } & \multicolumn{2}{|c|}{ misoginia } & \multirow{2}{*}{$\begin{array}{c}\text { TOTALES } \\
133\end{array}$} \\
\hline & 56 & $11,4 \%$ & 39 & $8,7 \%$ & 24 & $11,4 \%$ & 14 & $11,4 \%$ & \\
\hline evitativo & 43 & $7,9 \%$ & 18 & $3,7 \%$ & 13 & $4,5 \%$ & 15 & $6,5 \%$ & 89 \\
\hline ansioso & 56 & $4,9 \%$ & 22 & $2,6 \%$ & 36 & $7,3 \%$ & 30 & $4,1 \%$ & 144 \\
\hline desorganizado & 56 & $2,8 \%$ & 32 & $3,0 \%$ & 20 & $6,1 \%$ & 19 & $3,9 \%$ & 127 \\
\hline TOTALES & 211 & $27 \%$ & 111 & $18,1 \%$ & 93 & $29,2 \%$ & 78 & $15,8 \%$ & 493 \\
\hline
\end{tabular}

Fuente: elaboración propia. 
mencionan Ferrer y Bosh (2000). Los rasgos de masculinidad serían características deseables dentro de un espectro de expresión del género, haciendo énfasis en las diferencias masculinas y femeninas como separación de roles (Ferrer y Bosch 2000; Ferrer et al. 2004).

A partir de lo anterior, se desprende que los grupos de maltratadores ambulatorios podrían quedar fuera de la dicotomía machismomisoginia $\mathrm{v} / \mathrm{s}$ androginia-masculinidad, y estar mostrando una lucha contra las exigencias socioculturales expresadas en la cultura patriarcal, las que han sido internalizadas mediante un aprendizaje rígido e idiosincrático en cada sujeto, el cual reúne representaciones sociales e imágenes colectivas sobre lo deseable y lo indeseable respecto a ambos géneros y en su relación de pareja (De Keijzer 2003; Aguilera 2004; Escudero et al. 2005).

Además, hay un consenso en la literatura al momento de atribuir a la cultura patriarcal ciertas condiciones dominantes, como son: que las mujeres deben someterse a la autoridad del hombre y depender de ellos económicamente, y que el mejoramiento de las condiciones sociofamiliares de la mujer no siempre ha contribuido a arreglar las relaciones al interior de la familia, sino que aumenta el descontento del hombre en relación a sus supuestos privilegios masculinos de subordinación y sometimiento de la pareja (Garda 2004). Esto implica hipotetizar que existe un costo emocional que el maltratador debe pagar por mantener roles masculinos rígidos, lo que le adjudica "virilidad" dentro de un sistema patriarcal.

Es así que un acercamiento psicológicamente sano hacia lo femenino, se vuelve una tarea difícil y deja a las parejas en una imposibilidad de encuentro de géneros. Por ello, el hombre misógino y machista utiliza estereotipos que lo acercan hacia la cosificación de la figura femenina, como "madre" o "reina" (en el caso de la hija y la madre de él), o como "puta" en el caso de todas las mujeres y su esposa (Madrigal 2010).

Es por esto que la penalización de la violencia es tan importante para los sujetos de bajo, mediano y alto riesgo, porque la pena jurídica sirve como control social o como denuncia explícita de que el comportamiento del sujeto es ilegal. Un alto porcentaje de maltratadores tiene dificultades en el reconocimiento de algunas formas de violencia (sobre todo la psicológica, la económica, entre otras) cuando ingresan a terapia, porque las consideran legítimas dentro de un contexto de relación de pareja (Martín 2007; Civit Bel et al. 2013).

Por todo lo anterior, los hallazgos encontrados a partir del IMAFE sugieren revisar la centralidad que los hombres otorgan a la misoginia como principal componente de la violencia de pareja, ya que los datos están mostrando que no existe una dicotomía tan evidente entre misoginia y androginia (Ferrer y Bosch 2000; Ferrer et al. 2004). Existe un alto nivel de solapamiento entre aspectos culturales y valoraciones de distintos países en relación con las creencias misóginas-andróginas hacia la mujer, y particularmente en Chile habría concordancia con estudios multiculturales que señalan que existen países con una mayor y menor expresión de la masculinidad y la tolerancia de género (Fernández y Vergara 1998; Moya et al. 2002). Estos estudios plantean que Chile estaría en un extremo de baja expresión de machismo y alta expresión de la femineidad en relación con otros países tales como Argentina, 
México o Italia, los que tendrían el mayor índice de expresión de la masculinidad versus Chile, Costa Rica y España (Fernández y Vergara 1998; Moya et al. 2002).

Sinembargo, hay diferencias entremaltratadores y no maltratadores, lo cual podría suponer una relación entre comportamientos misóginos y trastornos de personalidad, entendiendo que el trastorno de personalidad es un patrón rígido de comportamiento y una ritualización de las relaciones interpersonales. Por lo tanto, se concuerda con algunas investigaciones que plantean que las creencias y comportamientos misóginos estarían mostrando graves trastornos de personalidad y precarios modos de afrontamiento ante los conflictos con la pareja íntima (Alonso et al. 2013; Brasfield 2014).

\subsection{Estilos de apego en hombres maltratadores de su pareja íntima}

En relación con los estilos de apego en agresores, un resultado importante es que ambos grupos con estilos de apego ansioso $y$ desorganizado presentan un mayor porcentaje de agresores. Tienen características similares tales como la mantención del control, la intimidación y la contaminación afectiva de su pareja, y también están asociados a la desconfianza, la celotipia y la preocupación por la distancia de la pareja íntima (Babcock et al. 2000; Dutton 2006). El presente hallazgo manifiesta cómo los estilos de apego inseguros, del tipo desorganizado y ansioso, pueden ser visibles entre los sujetos que están en situación de separación, abandono o de recuperación de su pareja íntima. Para Backcook (2000), los estilos de apego ansioso y desorganizado se podrían considerar como la reactividad del sujeto frente a la posibilidad de lejanía de su pareja, como es el caso de los maltratadores voluntarios que se han presentado a tratamiento a los centros de atención por motivación propia, y que presentan un mayor nivel de culpa y sufrimiento por la situación en la cual están involucrados (Barría y Macchiavello 2012b).

Hay evidencia, como la planteada por Fonagy et al. (2010), de que los estilos de apego, sobre todo el ansioso y el desorganizado, se asemejan a los trastornos límites de la personalidad. Sin embargo, existe un grupo que sigue siendo difícil de pesquisar, como es el caso de los psicópatas subclínicos, que inventarios como Millon III no los detecta, y en su lugar los categoriza dentro de los narcisistas, histriónicos, antisociales y autodestructivos. Futuras investigaciones nos podrían orientar sobre cuáles serían los mecanismos psicológicos que pueden estar operando en ellos frente a su vinculación con su pareja, entendiendo que son sujetos crueles, intolerantes y pobres empáticamente (Gondolf 1996; Gondolf 1999, 2007; Fonagy et al. 2010; Pozueco et al. 2014).

Fonagy (2000) plantea que los sujetos con estilos de apego desorganizado son más ansiosos y, por lo tanto, híper-reactivos, por lo que están relacionados, como se señaló anteriormente, con los trastornos de personalidad límite, histriónicos, narcisistas y con dependencia. Ellos son más celotípicos, dependientes de su pareja y propensos a la violencia si se cuestiona su figura de apego (Fonagy 2000). De lo anterior se puede inferir que sí existe concordancia con los hallazgos sobre los estilos de apego ansioso y desorganizado, y que la principal manifestación de la violencia de pareja es el control coercitivo violento, con bajos niveles 
de violencia física, alto nivel de violencia psicológica, y un amedrentamiento y reacción desmedida frente a la demanda, separación y protesta de la mujer, lo que ha sido señalado por Jhonson (2005) como "terrorismo íntimo" (Johnson y Leone 2005; Gondolf 2007; Barría y Macchiavello 2012a).

Como se observó en Tabla 1, los estilos de apego en los grupos derivados de Tribunales de Familia (por violencia psicológica) y de Fiscalía (por violencia física) se distribuyen homogéneamente, lo que permite entrever una importante línea de investigación que es la violencia cronificada, donde fenomenológicamente se están mostrando dos evidentes rasgos: primero, de psicopatía subclínica o maltratadores instrumentales; y segundo, de trastornos de personalidad límite. Debemos señalar que son fenomenológicos, porque es el clínico quien evidencia en la entrevista el tipo de vinculación del sujeto y el tipo de trastornos de personalidad que puede presentar el maltratador, que inventarios como Millon III tiende a no pesquisarlos, o los categoriza en una serie de trastornos distintos, como se ha señalado anteriormente.

Un hallazgo importante a partir del ERC-2, junto con demostrar que existen diferencias significativas entre maltratadores y no maltratadores, es que el constructo puede apreciarse con total nitidez en los estilos de apego temeroso y ansioso que se concentran entre los sujetos que se han presentado voluntariamente a los programas, y que manifiestan su angustia frente a la situación de separación o abandono. Del mismo modo, en los sujetos derivados de Fiscalía por violencia física están concentrados los estilos de apego ansioso o preocupado.
En relación a las escalas de ERC-2, existe una tendencia que marca que los grupos de maltratadores de riesgo bajo, medio y alto presentan mayores índices en la escala de ansiedad, que podría explicar la reactividad e impulsividad del sujeto (Babcock et al. 2000; Ross y Babcock 2009). Llama la atención que los grupos de riesgo extremo no presentan diferencias significativas en la escala de ansiedad, por lo que se podría hipotetizar la existencia de un grupo "psicópata subclínico" que tiende a controlar la ansiedad, pero que está asociado a la violencia más grave y lesional (Dutton 2006; Ross y Babcock 2009).

En síntesis, tanto el estilo de apego ansioso como desorganizado podrían explicar el $70 \%$ de los rasgos acosadores (celotipía, hiperreactividad y preocupación desmedida) que presentan los maltratadores por violencia de género. Sin embargo, queda un grupo significativo en torno al cual se requieren investigaciones específicas, como es el caso de los obsesivos compulsivos y los psicópatas adaptados o subclínicos que no necesariamente son acosadores y tienden a mantener el control de sus emociones, pero actúan violentamente por acumulación, esto es, soportan la queja de la pareja hasta que explotan y la agreden violentamente (Barría y Macchiavello 2012b; Dutton y Haring 1999; Holtzworth-Munroe et al. 1997; Loinaz et al. 2012).

\section{Limitaciones, sugerencias y proyecciones clínicas}

Una de las limitaciones que se presentó con los instrumentos, sobre todo en el IMAFE, es que se cuestionan las validaciones de constructo y fiabilidad que se han llevado a cabo en po- 
blaciones, sobre todo, de estudiantes universitarios (Gil-González 2005; Gómez et al. 2012), las que pueden reflejar una alta deseabilidad social y la incompatibilidad de muestras, o se pueden estar haciendo atribuciones a variables sobredimensionadas, especialmente cuando se quiere extrapolar a otras poblaciones específicas, como son los maltratadores.

En relación con ERC-2 hubo limitaciones del uso práctico de la información. Los equipos consideraron que los datos provistos por la entrevista son más relevantes que la aplicación del inventario. Creen que, al ser la violencia una situación grave, los estilos de apego no aportarían información adicional, sino que podrían ser complementarios a instrumentos que midan trastornos de personalidad y psicopatología en general.

Pese a ello, ambos instrumentos y sus respetivos constructos teóricos pueden ser útiles para investigar la violencia. Es posible comparar estos hallazgos con otras muestras clínicas y subclínicas en ambientes carcelarios, hospitalarios y educacionales.

\section{Bibliografía}

Aguilera, A. 2004. "Características psicológicas del hombre golpeador de su compañera permanente, residente en la ciudad de Bucaramanga". MedUNAB 7 (20): 73-82.

Ansbro, M. 2008. "Using attachment theory with offenders". Probation Journal 55 (3): 231-244.

Babcock, J. et al. 2000. "Attachment, emotional regulation, and the function of marital violence: Differences between secure, preoccupied, and dismissing violent and nonviolent husbands". Journal of Family Violence 15 (4): 391-409.

Barría, J. y Macchiavello, A. 2012a. "Anatomía de los hombres que ejercen violencia hacia sus parejas: Primer levantamiento de datos para el diseño de un perfil" Psicologia.com 16. En línea, disponible en: http:// www.psiquiatria.com/bibliopsiquis/handle/10401/5511 (visitado el 20 de abril de 2015).

2012b. "Hombres que ejercen maltrato hacia su pareja: Un actor desconocido". De Familias y Terapias 33: 121-140.

2013. Aproximación a un modelo teórico de tipologías de hombres maltratadores. El hombre agresor de su pareja: un actor desconocido. En línea, disponible en: http://repositorio.uchile.cl/handle/2250/114808 (visitado el 20 de abril de 2015).

Bonino, L. 2003. "Masculinidad hegemónica e identidad masculina". Dossiers Feministes 6: 7-36.
Bosch, E. et al. 2014. "Itinerarios hacia la libertad: La recuperación integral de las víctimas de la violencia de género". Psicología y Salud 15 (1): 97-105.

Brasfield, R. 2014. "The absence of evidence is not the evidence of absence: The abusive personality as a disordered mental state". Aggression and Violent Behavior 19 (5): 515-522.

Brennan, K. A. et al. 1998. "Self-report measurement of adult attachment: An integrative overview". Attachment theory and close relationships. En Simpson, J. A. y Rholes, W. S. (Eds.). New York: Guilford Press. 46-76.

Civit Bel, N. et al. 2013. Análisis de la motivación al cambio en sujetos que han realizado un programa formativo en violencia doméstica. Barcelona: Centre d' Estudis Jurídics i Formació Especialitzada.

De Keijzer, B. 2003. "Hasta donde el cuerpo aguante: Género, cuerpo y salud masculina". Foro Internacional en Ciencias Sociales y Salud. La salud como derecho ciudadano: Perspectivas y propuestas desde América Latina. Lima, Perú.

DeKeseredy, W. y Dragiewicz, M. 2007. "Understanding the complexities of feminist perspectives on woman abuse". Violence against Women 13 (8): 874-884.

Dutton, D. et al. 1997. El golpeador: Un perfil psicológico. Buenos Aires: Paidós.

. y Haring, M. 1999. "Perpetrator personality effects on post-separation victim reactions in abusive 
relationships". Journal of Family Violence 14 (2): 193-204. 2006. The abusive personality: Violence and control in intimate relationships. New York: Guilford Press.

Echauri, J. et al. 2005. "Teoría y descripción de la violencia doméstica: Programa terapéutico para maltratadores del ámbito familiar en el centro penitenciario de Pamplona". Anuario de Psicología Jurídica 15: 67-95.

Escudero, A. et al. 2005. "La persuasión coercitiva, modelo explicativo del mantenimiento de las mujeres en una situación de violencia de género I: Las estrategias de la violencia". Revista de la Asociación Española de Neuropsiquiatría 25 (95): 85-117.

Fernández, I. y Vergara, A. 1998. "La dimensión de masculinidad-feminidad y los antecedentes, las reacciones mentales y los mecanismos de autocontrol emocional". Revista de Psicología Social 13 (2): 171-179.

Ferrer, V. y Bosch, E. 2000. "Violencia de género y misoginia: Reflexiones psicosociales sobre un posible factor explicativo". Papeles del Psicólogo 75: 13-19. et al. 2004. "Estudio meta-analítico de características diferenciales entre maltratadores y no maltratadores: El caso de la psicopatología y el consumo de alcohol o drogas". Psykhe 13 (1): 141-156.

Fonagy, P. 2000. "Apegos patológicos y acción terapéutica". Aperturas Psicoanalíticas: Revista de Psicoanálisis 4 (4). En línea, disponible en: http://www. aperturas.org/articulos.php? $\mathrm{id}=0000104 \& \mathrm{a}=$ Apegospatologicos-y-accion-terapeutica (visitado el 20 de abril de 2015).

et al. 2010. Attachment and personality pathology. Psychodynamic psychotherapy for personality disorders (A clinical handbook). Washington, DC-London: American Psychiatric Publishing.

Fraley, Ch. et al. 2000. "An item response theory analysis of self-report measures of adult attachment". Journal of Personality and Social Psychology 78 (2): 350365.

Garda, R. 2004. "Complejidad e intimidad en la violencia de los hombres. Reflexiones en torno al poder, el habla y la violencia hacia las mujeres. Violencia Contra la Mujer en México 119. En línea, disponible en: http://200.4.48.30/webmujeres/biblioteca/Violencia/ Violencia\%20contra\%20la\%20mujer.pdf (visitado el 20 de abril 2015).

Gil-González, D. 2005. Relación entre androginia y bienestar psicológico en estudiantes universitarios. Tesis para optar al grado de Psicología. Puebla: Universidad de Las Américas.

Goldstein, H. y Higgins-D'Alessandro, A. 2000. "Empathy and attachment in relation to violent vs. non- violent offense history among jail inmates. Journal of Offender Rehabilitation 32 (4): 31-53.

Gondolf, E. 1996. Characteristics of batterers in a multisite evaluation of batterer intervention systems. En línea, disponible en: http://www.mincava.umn.edu/documents/ gondolf/batchar.pdf (visitado el 4 de marzo de 2015). 1999. "MCMI-III results for batterer program participants in four cities: Less "pathological" than expected". Journal of Family Violence 14 (1): 1-17. 2007. "Theoretical and research support for the Duluth Model: A reply to Dutton and Corvo". Aggression and Violent Behavior 12 (6): 644-657.

Holtzworth-Munroe, A. y Stuart, G. 1994. "Typologies of male batterers: Three subtypes and the differences among them". Psychological Bulletin 116 (3): 476-497. et al. 1997. "Violent versus nonviolent husbands: Differences in attachment patterns, dependency, and jealousy". Journal of Family Psychology 11 (3): 314-331.

Johnson, M. y Leone, J. 2005. The differential effects of intimate terrorism and situational couple violence findings from the national violence against women survey". Journal of Family Issues 26 (3): 322-349.

Loinaz, I. 2009. Aproximación teórica y empírica al estudio de las tipologías de agresores de pareja: Análisis descriptivo de variables e instrumentos de evaluación en el centro penitenciario Brians-2. En línea, disponible en: http://datos.bne.es/edicion/a4831003.html (visitado el 20 de abril de 2015).

et al. 2010. "Tipología de agresores contra la pareja en prisión”. Psicothema 22: 106-111.

et al. 2012. "Estilo de apego, empatía y autoestima en agresores de pareja". Terapia Psicológica 30 (2): 61-70.

Madrigal, L. 2010. Los hombres asumimos nuestra responsabilidad: Las masculinidades en la prevención de la violencia de género. En línea, disponible en: http:// sistemadif.jalisco.gob.mx/cepavi/Material_didactico/ Masculinidades/Los\%20hombres\%20asumimos\%20 nuestra\%20responsabilidad.doc (visitado el 20 de abril 2015).

Millon, T. y Davis, R. 1998. Trastornos de la personalidad: Más allá del DSM-IV. Barcelona: Masson.

Moya, M. et al. 2002. "Masculinidad-feminidad y factores culturales". Revista Española de y Motivación y Emoción 3: 127-142.

Pears, K. y Capaldi, D. 2001. "Intergenerational transmission of abuse: A two generational prospective study of an at-risk sample". Child Abuse \& Neglect 25 (11): 1439-1461.

Pozueco, J. et al. 2014. "The psychopathic intimate 
partner batterer: A non-psychopathological profile". Anales de Psicología 30 (1): 25-36.

Rey, A. y César, A. 2008. "Habilidades pro sociales, rasgos de personalidad de género y aceptación de la violencia hacia la mujer, en adolescentes que han presenciado violencia entre sus padres". Acta Colombiana de Psicología 11 (1): 107-118.

Robinson, L. 2005. Investigating a batterer typology: the role of personality characteristics, attachment, and family of origin dynamics. En línea, disponible en: http:// repository.tamu.edu/handle/1969.1/2458 (visitado el 20 de abril 2015).

Ross, J. y Babcock, J. 2009. "Proactive and reactive violence among intimate partner violent men diagnosed with antisocial and borderline personality disorder". Journal of Family Violence 24 (8): 607-717.

Stith, S. et al. 2000. The intergenerational transmission of spouse abuse: A meta-analysis. Journal of Marriage and Family 62 (3): 640-654.

Straus, M. et al. 1996. "The revised conflict tactics scales (CTS2) development and preliminary psychometric data". Journal of Family Issues 17 (3): 283-316.

Teruelo, J. 2011. "Feminicidios de género: Evolución real del fenómeno, el suicidio del agresor y la incidencia del tratamiento mediático". Revista Española de Investigación Criminológica 9: 1-27. 
